\title{
Redefinition of tricuspid valve structures for successful ring annuloplasty
}

\author{
Noriyasu Kawada, MD, ${ }^{\text {a }}$ Hirokuni Naganuma, $\mathrm{MD}, \mathrm{PhD},{ }^{\mathrm{a}}$ Koichi Muramatsu, MD, \\ Hatsue Ishibashi-Ueda, $\mathrm{MD}, \mathrm{PhD},{ }^{\mathrm{b}}$ Ko Bando, $\mathrm{MD}, \mathrm{PhD},{ }^{\mathrm{c}}$ and Kazuhiro Hashimoto, $\mathrm{MD}, \mathrm{PhD}^{\mathrm{c}}$
}

\section{ABSTRACT}

Background: Although numerous reports have described suturing techniques for tricuspid annuloplasty, most studies were not based on a detailed anatomy of the tricuspid annulus. Thus, the definition of the tricuspid commissures remains unclear. This study aimed to clearly define the commissures and leaflets of the tricuspid valve and subvalvular structures, and to define a standard method for tricuspid annuloplasty.

Methods: In 27 normal heart specimens without cardiac disease, the tricuspid commissure was defined using indentations of the leaflets as a point, not an area, and the length of each tricuspid annulus was measured. The relationships between the leaflets and the subvalvular structures were then examined.

Results: In most specimens, the posterior leaflet had $2(62.9 \%)$ or $3(29.6 \%)$ scallops, providing further evidence of posterior leaflet diversity. In addition, the posterior leaflet had 1 or 2 indentations, which can be mistaken for true commissures. The annulus of the posterior leaflet was significantly longer than the annuli of the other 2 leaflets $(P<.00428)$. The annuli of the septal and the anterior leaflets were supported by the interventricular septum and the supraventricular crest, respectively, whereas the posterior leaflet annulus was distributed largely along the right ventricular free wall.

Conclusions: There was a structural gap between the tricuspid leaflet indentations and the subvalvular structures. The relationships among the leaflets, commissures, and subvalvular structures differed in the septal, anterior, and posterior leaflets. This new definition of the commissural point may aid the development of a clear-cut methodology for prosthetic ring annuloplasty. (J Thorac Cardiovasc Surg 2018;155:1511-9)

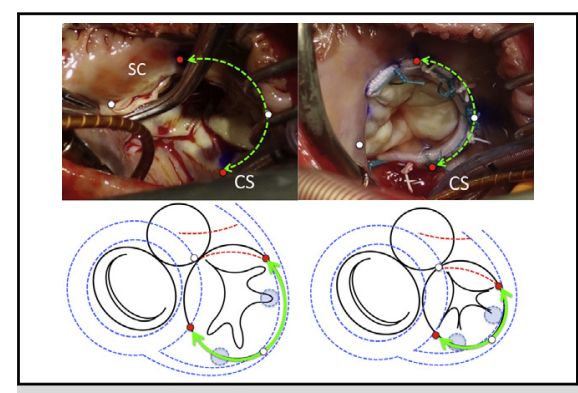

Based on the relationship of the subvalvular structures, the enlargement of the tricuspid annulus will spread from the posterior leaflet.

\section{Central Message}

This new definition of the commissural point may aid the development of a clear-cut methodology for prosthetic ring annuloplasty.

\section{Perspective}

The definition of the tricuspid commissures remains unclear. By defining the commissure as a point, the tricuspid valve leaflets can be clearly distinguished. We need to rethink the relationships of the leaflets, commissures, and subvalvular structures. This new definition of the commissural point may aid the development of a clear-cut methodology for prosthetic ring annuloplasty.

See Editorial Commentary page 1520.
Surgical treatment has become an optimal modality for early intervention of tricuspid regurgitation (TR) secondary to left heart pathology that results in volume overload, such as mitral valve regurgitation. ${ }^{1}$ Previous studies have shown that the main TR pathology results from enlargement of the

\footnotetext{
From the ${ }^{a}$ Department of Cardiovascular Surgery, Jikei University Kashiwa Hospital, Chiba, Japan; ${ }^{\mathrm{b}}$ Department of Pathology, National Cerebral and Cardiovascular Center, Osaka, Japan; and ${ }^{\mathrm{c}}$ Department of Cardiovascular Surgery, Jikei University, Tokyo, Japan.

Received for publication July 29, 2017; revisions received Nov 20, 2017; accepted for publication Dec 11, 2017; available ahead of print Feb 1, 2018.

Address for reprints: Noriyasu Kawada, MD, Department of Cardiovascular Surgery, Jikei University Kashiwa Hospital, 163-1 Kashiwashita-Kashiwa City, Chiba 277-8564, Japan (E-mail: nkwd@cf6.so-net.ne.jp). $0022-5223 / \$ 36.00$

Copyright (C) 2017 by The American Association for Thoracic Surgery https://doi.org/10.1016/j.jtcvs.2017.12.045
}

annulus. ${ }^{2}$ Based on the Society of Thoracic Surgeons National Database, the most common type of repair is tricuspid valve annuloplasty only. ${ }^{3}$ Long-term studies of tricuspid valve repair have shown that prosthetic ring annuloplasty is more durable than suture annuloplasty. ${ }^{4,5}$

Various models of tricuspid annuloplasty rings are currently available. In the early 1970s, tricuspid rings were typically formed in a single plane and differed from the actual nonplanar or 3-dimensional valve geometry reported

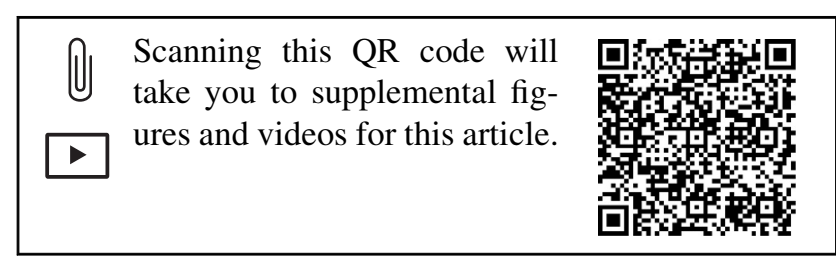




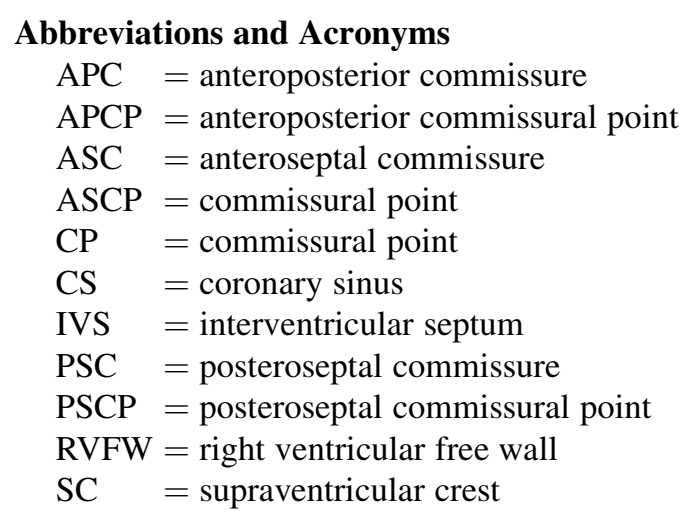

in several recent studies using real-time 3-dimensional echocardiography to assess healthy human tricuspid valves. ${ }^{6,7}$ Consequently, newer versions of the tricuspid ring with a 3-dimensional design were introduced in the late 1990s. In addition, markers on the rings are defined as points, not as areas, and the distances between the markers vary among manufacturers. Thus, the gold standard technique for tricuspid ring annuloplasty remains controversial. Although there is an ongoing discussion on whether flexible or rigid prosthetic rings should be used in tricuspid valve repair, no report to date has addressed a detailed and specific methodology for ring annuloplasty. ${ }^{8}$ Therefore, in this study we reexamined the anatomy of the tricuspid valve in normal heart specimens with an eye to standardizing the surgical procedure for tricuspid ring annuloplasty.

\section{METHODS}

Jikei University's Institutional Review Board approved this anatomic study (approval no.28-128 [8371]).

\section{Definitions and Assessments}

The length of the tricuspid annulus was measured in 27 normal heart specimens that were free of valvular disease based on the following definitions of leaflets. The anteroseptal commissural point (ASCP) closest to the indentation of the annulus located on the anteroseptal commissure (ASC) region in which the commissure could be easily identified is designated the primary reference point. Subsequently, based on the characterization by Silver and colleagues" that "...the tricuspid valve has an anterior leaflet, a posterior leaflet with a variable number of scallops, and a single septal

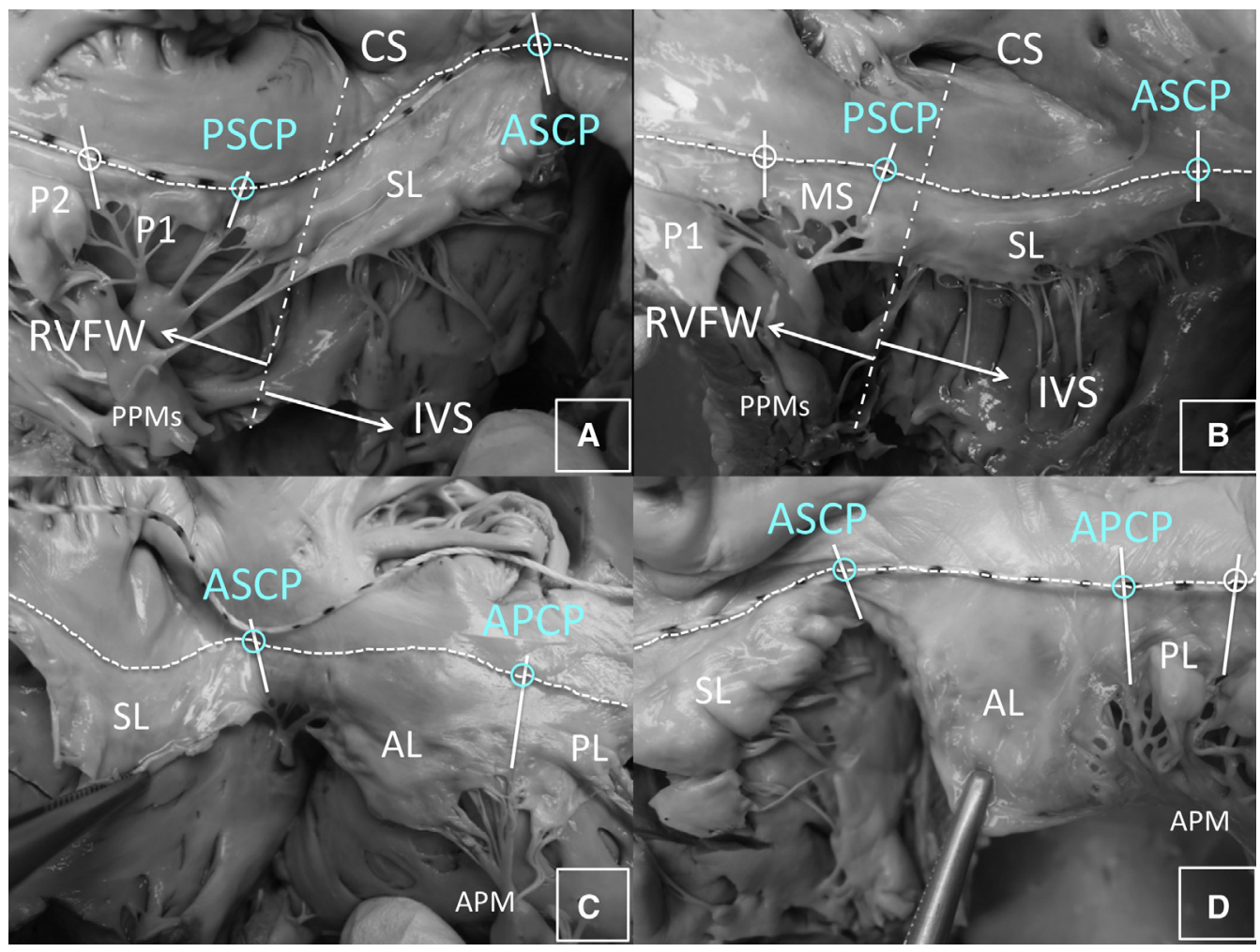

FIGURE 1. Definition of the leaflets. A and B, The indentation to which the first fan-shaped chord toward the septal leaflet from the anteroseptal commissure (ASC) is attached is designated the posteroseptal commissural point (PSCP). The PSCP is located on the free wall. The commissure of posterior leaflet 1 $(P 1)$ and posterior leaflet $2(P 2)$ is located on the right ventricular free wall ( $R V F W$ ). As shown, if the folding point of the RVFW (chain line) cannot be identified during cardiotomy, the coronary sinus $(C S)$ may be serve as another key feature to guide positioning. $\mathrm{C}$ and $\mathrm{D}$, The indentation to which the first fan-shaped chord toward the anterior leaflet $(A L)$ from the ASC is attached is designated the anteroposterior commissural point $(A P C P)$. The shape of the AL is relatively uniform, making it easy to distinguish. $A S C P$, Anteroseptal commissural point; $S L$, septal leaflet; $P P M$, posterior papillary muscle; IVS, interventricular septum; $M S$, mini-scallop; $P L$, posterior leaflet; $A P M$, anterior papillary muscle. 


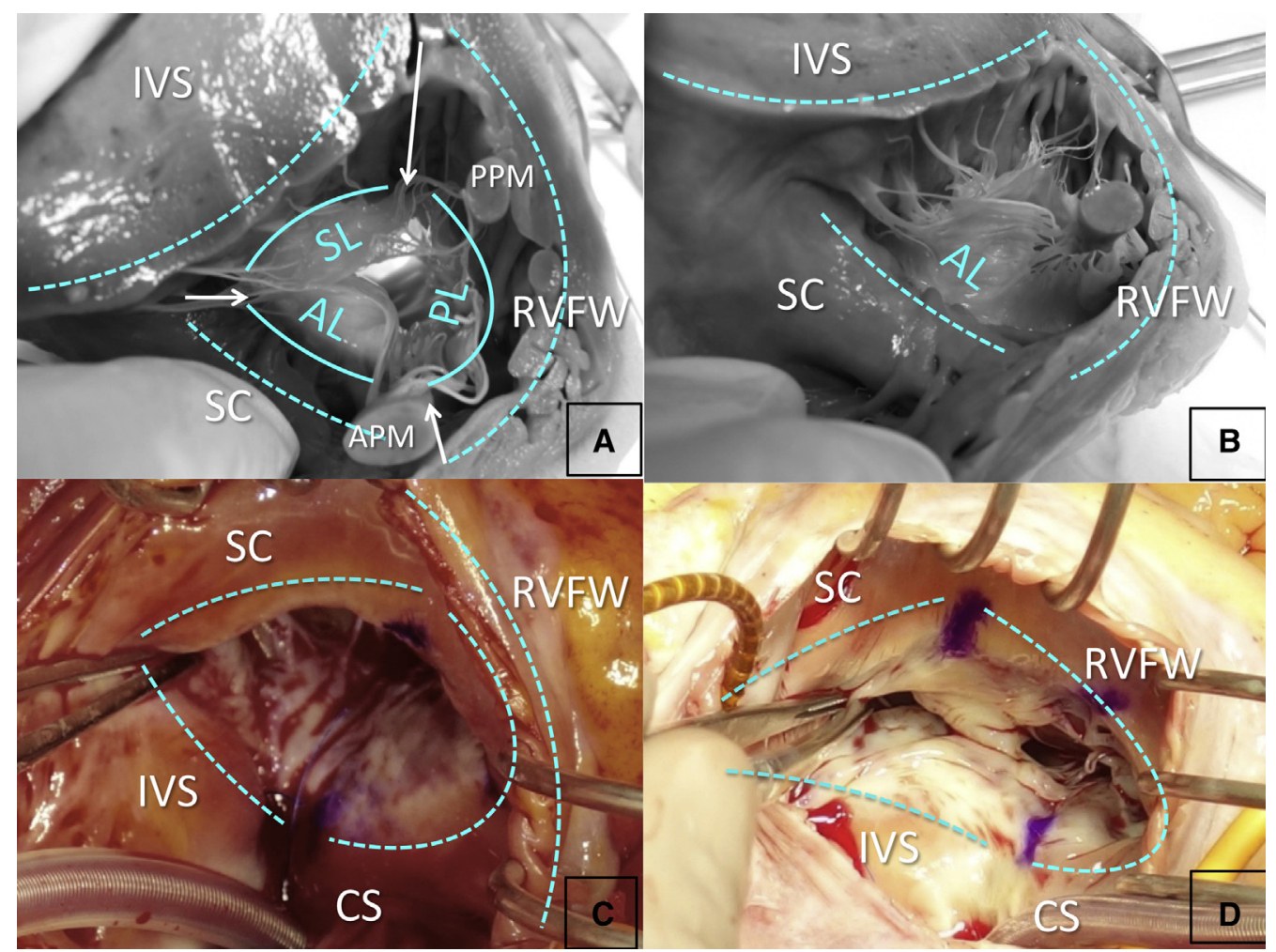

FIGURE 2. Relationship between the tricuspid annulus and the subvalvular apparatus in a cadaveric heart and the intraoperative view. A and B, In a cadaveric specimen, much of the septal leaflet $(S L)$ originates from the interventricular septum (IVS), and a part of this leaflet connects to the right ventricular free wall $(R V F W)$. The posterior leaflet $(P L)$ is diverse in shape and originates from the RVFW. Much of the anterior leaflet $(A L)$ annulus is supported by the supraventricular crest $(S C)$, which has muscle bundles extending in the other direction. Indeed, the right ventricle is D-shaped, but the tricuspid annulus shape is circular, as shown on 3D echocardiography. C and D, Intraoperative view of the folding point on the SL toward the RVFW, as well as that on the anterior wall from the SC to the RVFW. The enlargement of the posterior leaflet annulus appears to be greater in patients with high-grade tricuspid regurgitation (TR). C, Mild TR. D, Severe TR. PPM, Posterior papillary muscle; APM, anterior papillary muscle; CS, coronary sinus.

leaflet," the indentation to which the first fan-shaped chord toward the septum from the foregoing ASC is attached is designated the posteroseptal commissure (PSC) (Figure 1). Thus, the cusp located between the ASC and PSC is defined as the septal leaflet. Similarly, the indentation to which the first fan-shaped chord toward the front from the ASC is attached is designated the anteroposterior commissure (APC). Accordingly, the cusp located between the ASC and APC is defined as the anterior leaflet. The entire cusp between the PSC and APC, including the remainder of all indentations, scallops, and accessory leaflets varying in number and shape, is defined as the posterior leaflet. ${ }^{9}$ The commissures are identified as the continuity with the 2 major papillary muscles, the anterior and posterior papillary muscles, and the spatial relationship between the opening of the coronary sinus (CS), and folding around the interventricular septum (IVS) and the free wall is seen. Finally, the point closest to the annulus from the edge of the indentation of the PSC or APC is defined as the commissural point $(\mathrm{CP})$.

In most specimens, the PSC point was located on the right ventricular free wall (RVFW). The APC could be easily identified, and the reproducibility was as high as that of the ASC (Figure 1).

Measurements of the annuli were performed to obtain the length of the annuli using the $3 \mathrm{CPs}$ as defined above. Because the tricuspid annulus has a 3-dimensional structure, a silk thread was placed along the annulus to mark the positions before taking the measurements (Figure 1).

Based on these definitions, we assessed the relationships between the CPs and subvalvular anatomic structures.

\section{Statistical Analysis}

Statistical analyses were performed with Excel version 14.7.0 (Microsoft, Redmond, Wash) using the add-in software StatMate V (ATMS, Tokyo, Japan). Because the data in all groups showed a normal distribution, 1 -way analysis of variance was used for the 3-group comparison.

\section{RESULTS}

The posterior leaflets in the 27 normal heart specimens had 1 scallop in 1 specimen (3.7\%), 2 scallops in 17 specimens $(62.9 \%), 3$ scallops in 8 specimens $(29.6 \%)$, and 4 scallops in 1 specimen $(3.7 \%)$. Most specimens had 2 scallops, with a wide variety of shapes. The mean annulus length was $32.3 \pm 7.1 \mathrm{~mm}$ for the septal leaflets, $32.1 \pm 4.7 \mathrm{~mm}$ for the anterior leaflets, and $41.4 \pm 8.4 \mathrm{~mm}$ for the posterior leaflets. Thus, the annulus of the posterior leaflet was significantly longer than the annuli of the other 2 leaflets (3-group comparison; $P=.00428)$. The annuli between the ASCP and posteroseptal commissural point (PSCP) were located close to the IVS and were supported securely. Because of the fan-shaped chord extending from the posterior papillary muscle 


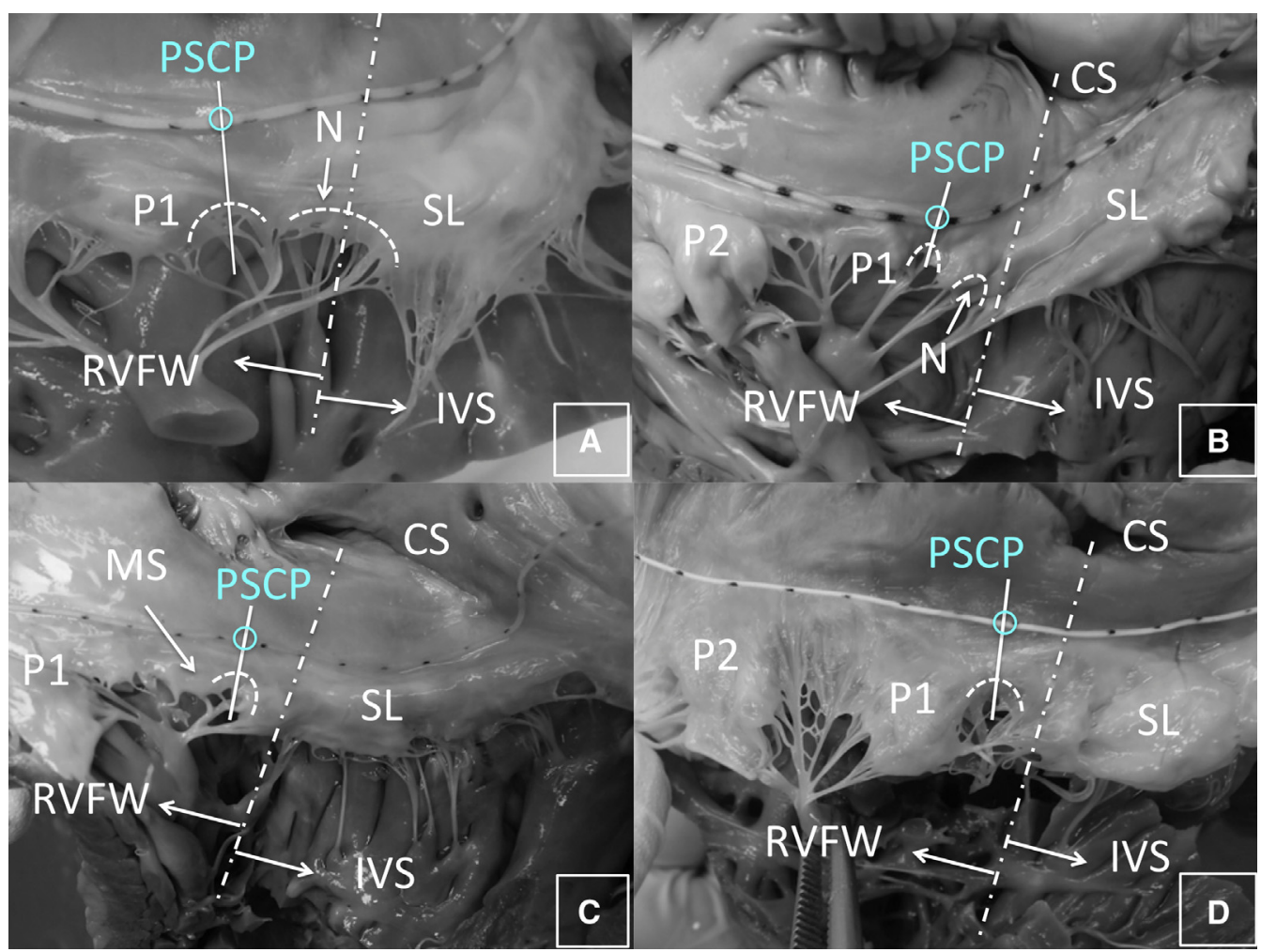

FIGURE 3. Variation of the posteroseptal commissure (PSC) region. A, PSC with wide notch. B, PSC with narrow notch. C, PSC without notch, with miniscallop. D, PSC without notch and miniscallop. As shown, the PSC region is diverse in shape (A-D); however, the posteroseptal commissural point $(P S C P)$, determined based on the definitions proposed herein is always located on the right ventricular free wall $(R V F W)$. The coronary sinus $(C S)$ has a key function in verifying the border between the RVFW and the interventricular septum (IVS). A and B, The notch is located further away from the annulus compared with the other indentations. The white dashed-and-dotted line indicates the border between the RVFW and the IVS. The white line represents the PSC line; the blue circle, the PSCP. SL, Septal leaflet; $P 1$, posterior leaflet $1 ; N$, notch; $P 2$, posterior leaflet $2 ; M S$, miniscallop.

continued to the PSC in most cases, the PSCP was located on the RVFW rather than on the IVS (Figures 1-3). The annuli between the ASCP and anteroposterior commissural point (APCP) were located almost on the supraventricular crest (SC), and they were supported securely. The annuli between the PSCP and APCP were located close to the RVFW (Figures 2 and 4).

Our findings confirm previous reports ${ }^{9}$ of the presence of scallops of varying number and shape in the posterior leaflets. The PSC region can be disorienting, consisting of such features as accessory leaflets, mini-scallops, notches, and posterior leaflets (Figure 3).

\section{DISCUSSION}

Cardiovascular surgeons often refer to the milestone paper on Carpentier's technique of tricuspid valve repair using suitably shaped and sized prosthetic rings. ${ }^{10}$ This method is derived from work by Deloche and colleagues,${ }^{11}$ who proposed the concept of the commissural leaflet, which defines a commissure as an area, not a single point. Annuloplasty using a prosthetic ring is the most common technique for tricuspid valve repair $^{3,12}$; however, currently available prosthetic tricuspid valve rings are marked only with points at the commissures, and it is not clear which part of the commissural leaflet is to be aligned with the marked point. In addition, the ring manufacturers note that the marked point is to be used only as an approximation.

In the present study, we took the characterization by Silver and colleagues ${ }^{9}$ into consideration when defining the reference points to achieve reproducibility. We propose that there is only 1 septal leaflet (the first indentation from the ASC toward the IVS) and only 1 anterior leaflet (the first indentation from the ASC toward the anterior aspect); all other indentations are part of the posterior leaflet.

Thus, as a new concept, we propose more straightforward, standardized definitions of the anatomy of the tricuspid valve with introduction of the CP (Figures 4 and 5). In accordance with the characterization of the tricuspid valve as consisting of 3 leaflets, we reevaluated its features with a focus on the length of the annulus of each leaflet. Consequently, we found the posterior leaflet annulus to be significantly longer than the other 2 leaflet annuli. Although this finding is not consistent with the findings reported by Deloche and associates, ${ }^{11}$ the region designated the commissural leaflet by those authors was in effect included as part of the posterior leaflet in our study. 


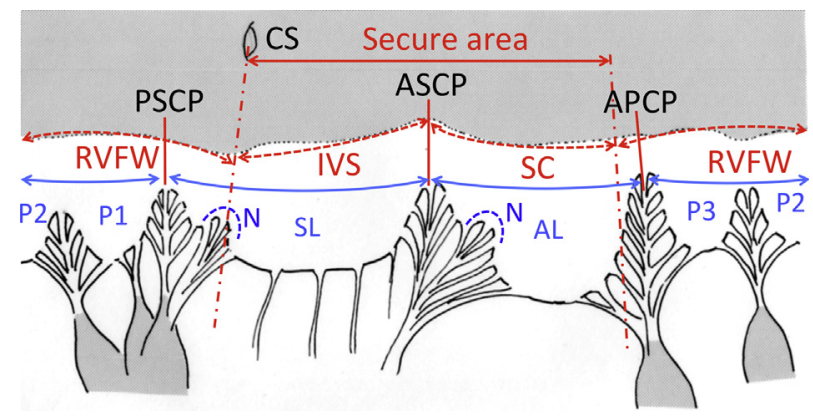

FIGURE 4. Schematic diagram of the tricuspid valve and subvalvular structures. The anterior leaflet $(A L)$ annulus is supported mostly by the supraventricular crest $(S C)$, and the septal leaflet $(S L)$ originates mostly from the interventricular septum (IVS). In contrast, the posterior leaflet (PL) originates from the free wall. Based on the subvalvular structures, changes to the IVS and SC are considered unlikely; therefore, the enlargement of the annulus would spread from the PL, which is continuous with the free wall. The solid red line represents each tricuspid leaflet, defined by the commissural point (CP); the dotted line represents the annular line, defined by subvalvular structures; and the dashed-dotted line represents the secure area consisting of the IVS and SC. CS, Coronary sinus; PSCP, posteroseptal commissural point; $A S C P$, anteroseptal commissural point; $A P C P$, anteroposterior commissural point; $R V F W$, right ventricular free wall; $P 1$, posterior leaflet $1 ; P 2$, posterior leaflet $2 ; N$, notch; $P 3$, posterior leaflet 3 .

Athavale and colleagues ${ }^{13}$ recently published detailed anatomic studies of the tricuspid valve. They described the inferior (posterior) leaflet as showing variations ranging from an independent leaflet to apportioned with the anterior or septal leaflet completely or partially. This resulted in identification of indistinct minor commissures. On close examination of this region, the PSC point was clearly located on the free wall in most cases, even when determined based on the criteria proposed in this study. Similarly, according to Seccombe and colleagues, ${ }^{14}$ it is not unusual for the septal leaflet to "wrap around" onto the posterior portion of the free wall by as much as $1 \mathrm{~cm}$.

Victor and Nayak ${ }^{15}$ assumed that "tricuspid is bicuspid," which is an oversimplification of a highly variable valve. They pointed out that the veil of valve leaflet tissue hanging down from a D-shaped annulus consists of straight (septal) and curved (nonseptal or mural) components. Because the right ventricle is collapsed during surgery in arrest, and at the time of examination in the cadaveric hearts, the annulus contour differs from that of the normal cardiac beating cycle. The normal tricuspid valve closes in a trifoliate fashion, and the tricuspid annulus is elliptical or nearly circular on 3-dimensional echocardiography, and the right ventricle is D-shaped. ${ }^{7}$ The indentation between posterior leaflets 1 and 2 may resemble the PSC, which can lead to misjudgment (Figure 6).

We speculate that such misjudgment during surgery results from a lack of effort to carefully examine the subvalvular structures in relation to the PSC, while the definitions of the tricuspid valve, including the PSC, remain

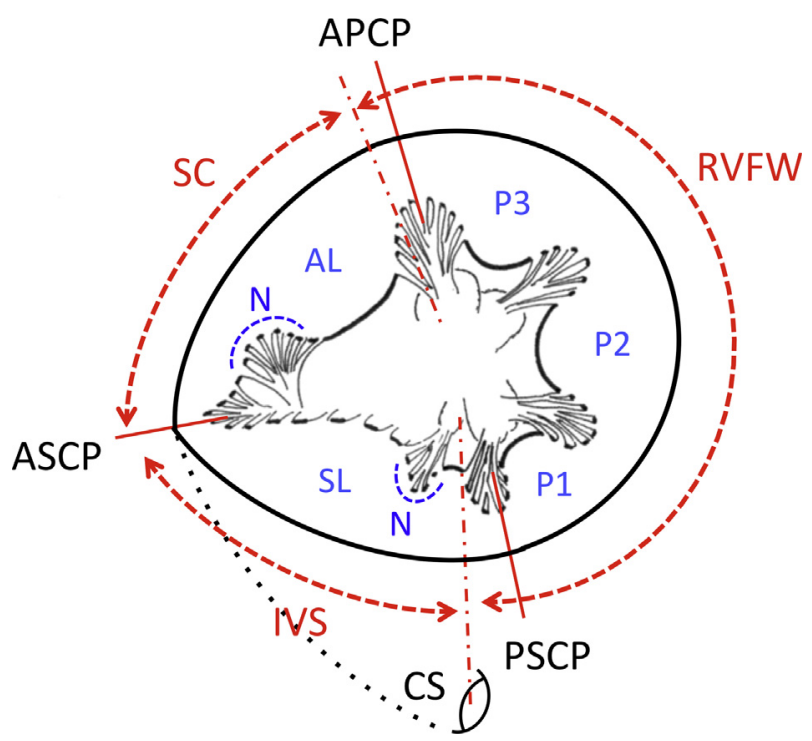

FIGURE 5. Schematic diagram of the surgeon's view of the tricuspid valve. The posterior leaflet annulus should be as long as the other 2 leaflet annuli. APCP, Anteroposterior commissural point; $S C$, supraventricular crest; $R V F W$, right ventricular free wall; $N$, notch; $A L$, anterior leaflet; $P 1$, posterior leaflet $1 ; P 2$, posterior leaflet $2 ; P 3$, posterior leaflet 3 ; $A S C P$, anteroseptal commissural point; $S L$, septal leaflet; IVS, interventricular septum; $P S C P$, posteroseptal commissural point; $C S$, coronary sinus.

ambiguous. Thus, part of the posterior leaflet may be mistaken for the septal leaflet, and this area may be further misinterpreted to include the region that is shifted to the RVFW with frequent movements. Consequently, excess tension may be placed around the septal leaflet and a part of the posterior leaflet during surgery when the suture is passed around the prosthetic ring, making the tricuspid valve shape disproportional to its original anatomy. It is possible that these mistakes account for the reports describing ring detachment from the septal leaflet as a downside to using rigid rings or for the emergence of theory-like septal plication. ${ }^{16,17}$

The anatomic principles forming the basis of ring annuloplasty are believed to depend on the relationship between the subvalvular structures and the annulus, as in the case of the mitral valve. Based on the definitions proposed herein, our findings show that the septal leaflet originates mostly from the IVS. Conversely, the posterior leaflet takes diverse shapes and originates from the free wall. Examining the tricuspid annulus from the right ventricle shows that the anterior leaflet annulus is supported mostly by the SC, which has muscle bundles extending in the other direction. ${ }^{18}$ The $\mathrm{SC}$ has a function similar to that of the adductor muscle of scallops in connecting the RVFW to the IVS, and is continuous with the trabecula septomarginalis.

Victor and Nayak ${ }^{15}$ showed that the mural leaflet is a leaflet from the free wall, but the anterior leaflet originates 


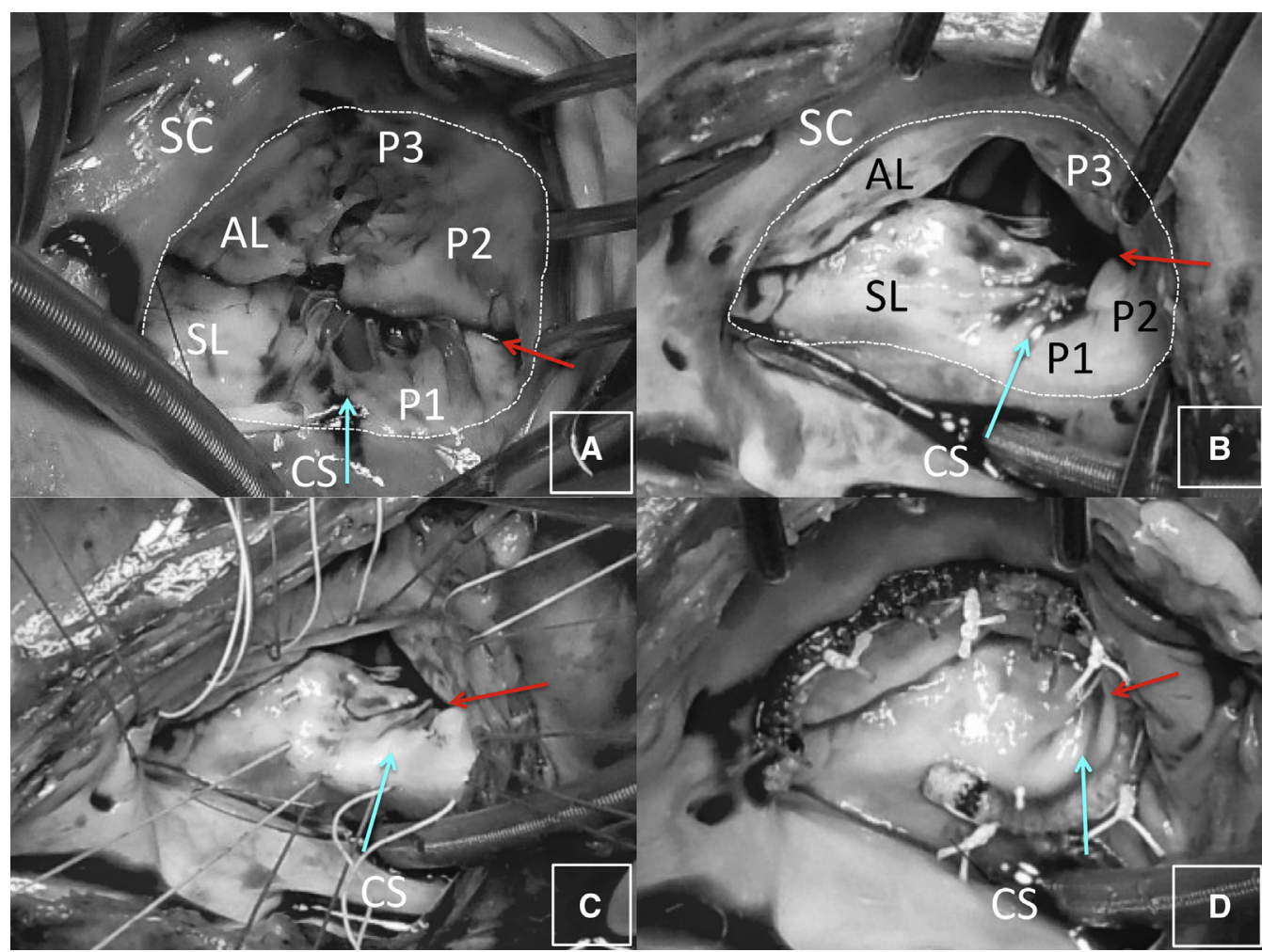

FIGURE 6. Misjudgment of the posteroseptal commissure (PSC). A and B, Due to right ventricular collapse during surgery, the tricuspid valve changes in shape depending on how the needle is pulled. The PSC is particularly difficult to identify, and the area between P1 and P2 or that between P2 and P3 is often mistaken for the posteroseptal commissural point (PSCP). C and D, Using a flexible band (SJM Talor band; St Jude Medical, St. Paul, Minn), the true PSCP (blue arrow) and the false PSCP (red arrow) were identified. SC, Supraventricular crest; $A L$, anterior leaflet; $S L$, septal leaflet; $P 1$, posterior leaflet 1; $P 2$, posterior leaflet $2 ; P 3$, posterior leaflet $3 ; C S$, coronary sinus orifice.

from the SC, which does not originate from the free wall. According to Buckberg and colleagues, ${ }^{19}$ the IVS is the "lion of right ventricular function," based on the structure of myocardial fibers. The RVFW is most susceptible to amplification of secondary TR before an apparent decrease in right heart function. Based on the structures, changes to

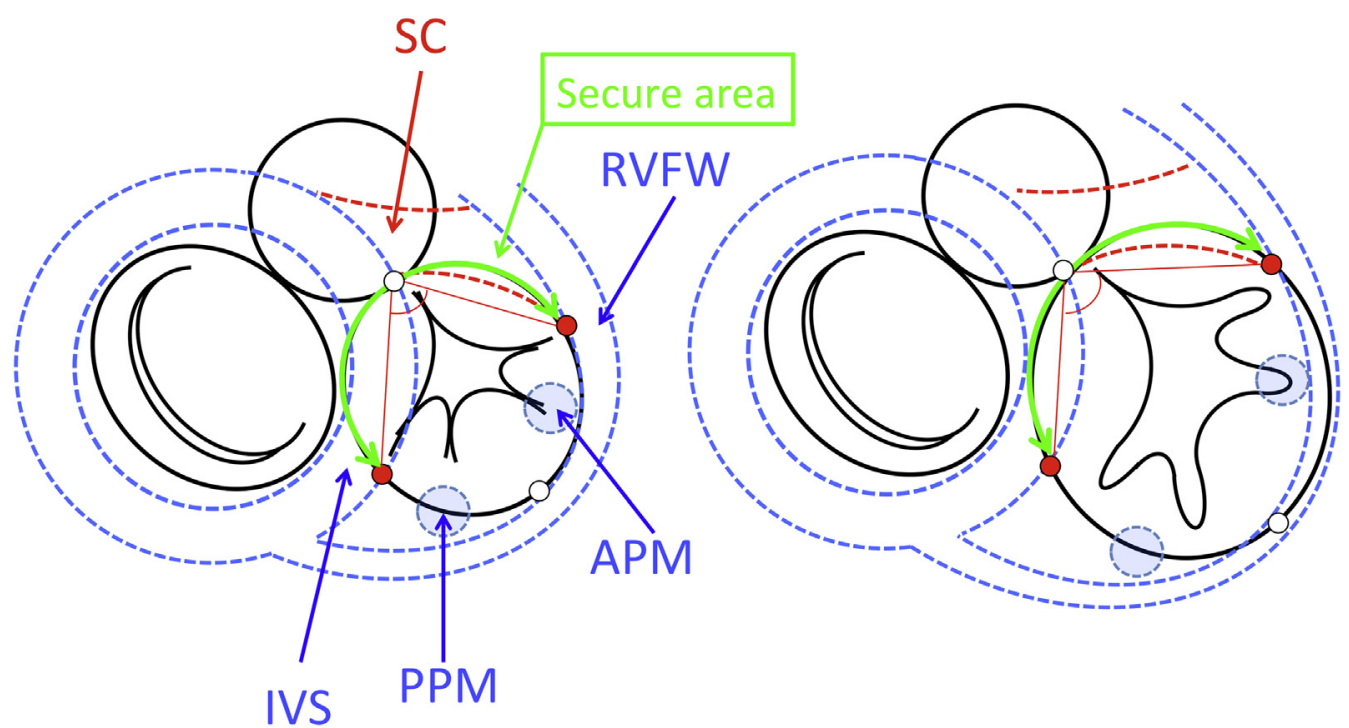

FIGURE 7. Annulus enlargement. Adding the subvalvular structure to the figure of annulus enlargement published by Ton-Nu and colleagues, ${ }^{20}$ it becomes clear that the annulus enlargement is centered around the posterior leaflet. This also suggests that the nature of the saddle shape is associated with the folding toward the free wall below the valve. Furthermore, the interventricular septum (IVS)-supraventricular crest (SC) angle will also become large as the right ventricular free wall $(R V F W)$ dilates. $A P M$, Anterior papillary muscle; $P P M$, posterior papillary muscle. 


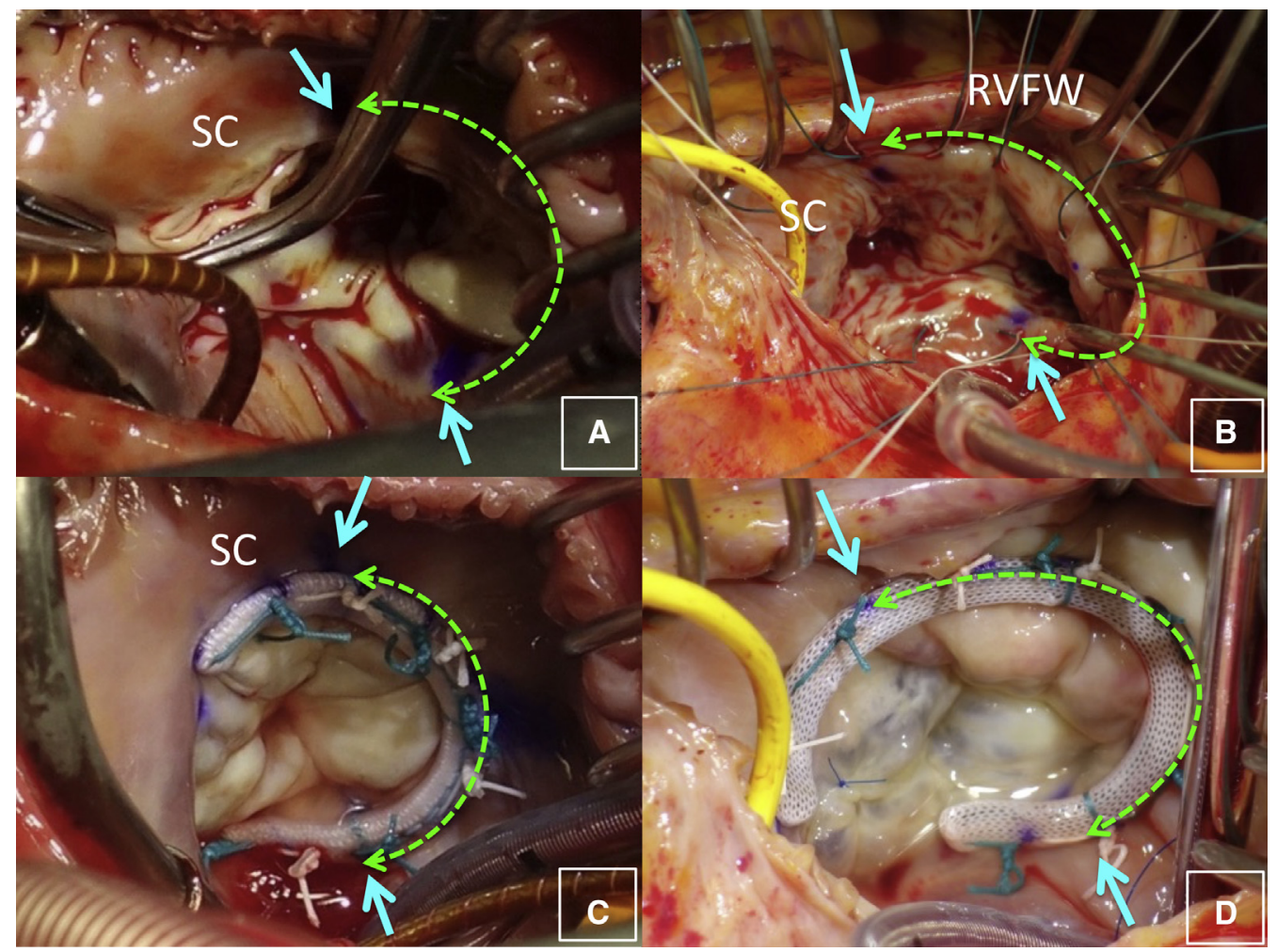

FIGURE 8. Current strategies for tricuspid valve annuloplasty. A, A patient with mild tricuspid regurgitation (TR). The two blue arrows each indicate folding points. B, The rigid area aligns with the right ventricular free wall $(R V F W)$, between the posteroseptal commissural point and the anteroposterior commissural point (APCP), which resembles the folding point on the septal leaflet toward the free wall, as well as that on the anterior wall from the supraventricular crest $(S C)$ to the free wall. C, A patient with severe TR. D, Markers on the rings are too close; the new point was rewritten 8-10 mm in the cranial direction for $8 \sim 10 \mathrm{~mm}$, which aligns the APCP on it.

the IVS and SC are considered unlikely. Therefore, enlargement of the annulus would spread from the posterior leaflet, which is continuous with the free wall (Figure 2). This view

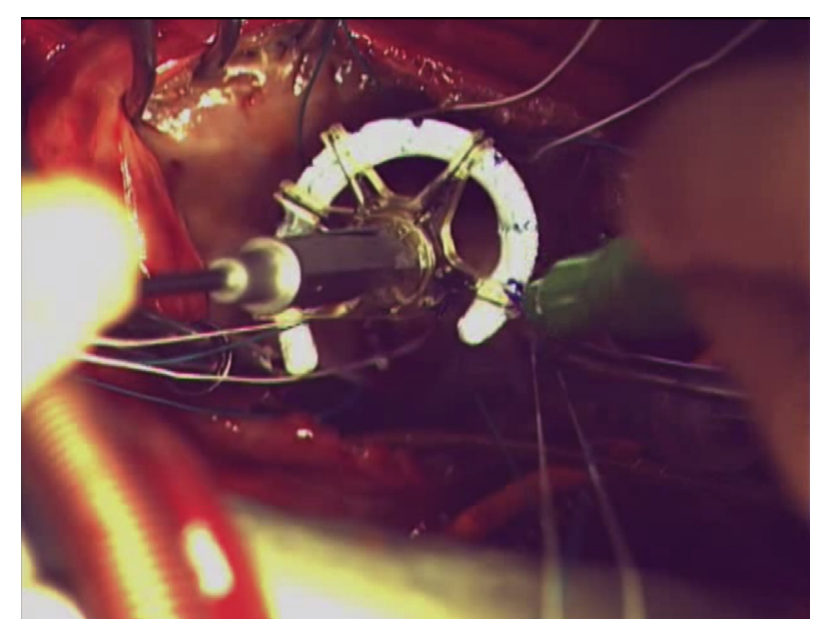

VIDEO 1. The current strategy for tricuspid valve annuloplasty in a patient with mild tricuspid regurgitation. Video available at: http://www. jtcvsonline.org/article/S0022-5223(17)32891-X/fulltext. was supported by an echocardiogram of the enlarged tricuspid annulus in a clinical case reported by Ton-Nu and colleagues. ${ }^{20}$ Those authors describe a feature called the lower point, which resembles the folding point on the septal leaflet toward the free wall, as well as that on the anterior wall from the SC to the free wall. The structural differences on the ventricular side are thought to make the tricuspid annulus form a saddle shape (Figures 2, 4 and 5). Victor and Nayak ${ }^{15}$ also described the curved leaflet as having a slit at the acute border, because the leaflet needs to angulate at this location during systole. This results in the curved leaflet being divided into "anterior" and "inferior" scallops, which along with the septal leaflet form a trileaflet valve, justifying the name "tricuspid valve." 21

In the present study we have introduced the concept of the $\mathrm{CP}$ and reexamined the lengths of the annuli. In addition, a specific definition of the commissure was proposed, and the definition of the posterior leaflet was reevaluated. Our present strategy for tricuspid valve annuloplasty is as follows: (1) mark 3 CPs, as described above; (2) assess the relationship between the CPs and subvalvular surrounding anatomic structures (eg, CS, SC, and membranous septum); (3) suture the mattress sutures through the tricuspid annulus 


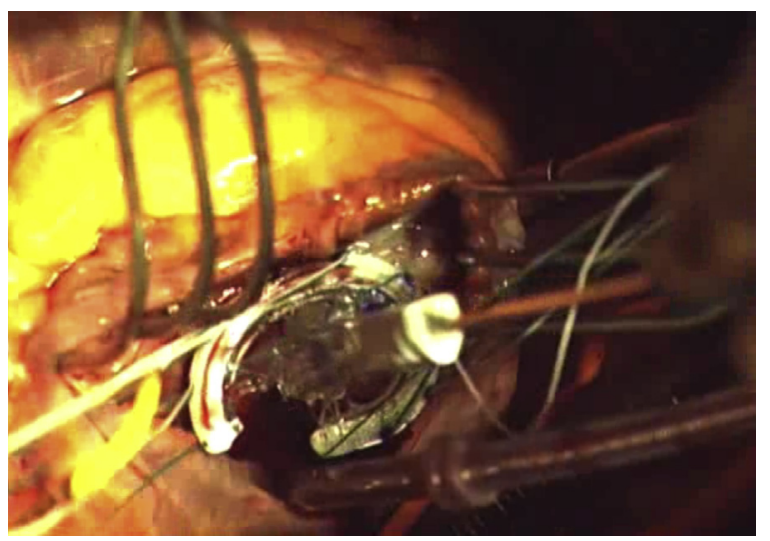

VIDEO 2. The current strategy for tricuspid valve annuloplasty in a patient with moderate to severe tricuspid regurgitation. Video available at: http://www.jtcvsonline.org/article/S0022-5223(17)32891-X/fulltext.

around the orifice, for a counterclockwise rotation, while avoiding the area of atrioventricular conduction tissue; (4) determine the length of the attachments of the tricuspid septal leaflet (between ASCP and PSCP); and (5) determine the APCP and PSCP positions on the ring.

For mild TR, we usually use the Tri-Ad ring (Medtronic, Minneapolis, Minn). The rigid area in this ring aligns the RVFW between the PSCP and APCP, which resembles the folding point on the septal leaflet toward the free wall, as well as that on the anterior wall from the SC to the free wall, because the tricuspid annular saddle shape is maintained (Figure 7, Figure 8, $A$ and $B$, and Video 1).

For moderate to severe TR, we usually use a 3D-shaped ring such as the Physio tricuspid ring (Edwards Lifesciences LLC, Irvine, Calif) or the Contour 3D ring (Medtronic). Because the markers on these rings are too close, a new point $8-10 \mathrm{~mm}$ in the cranial direction from original position is required. Then the APCP is aligned with the new point on these rings (Figure 7, Figure 8, $C$ and $D$, and Video 2).

We are currently conducting a clinical study in which the length of the tricuspid annulus is measured in patients with a functional TR during surgery according to the definitions proposed herein. The length of the anterior leaflet annulus or that of the septal leaflet annulus in these patients is no different from that measured in this study. However, the posterior leaflet annuli in the hearts of these patients appear to be larger than those in the normal hearts used in this study. The mean annulus length was $33.9 \pm 8.8 \mathrm{~mm}$ for the septal leaflets, $33.8 \pm 6.6 \mathrm{~mm}$ for the anterior leaflets, and $48.6 \pm 11.9 \mathrm{~mm}$ for the posterior leaflets (unpublished data). Moreover, enlargement of the posterior leaflet annulus seems to be greater in patients with high-grade TR (55.2 $\pm 13.5 \mathrm{~mm}$; unpublished data) (Figures 7 and 8). The measurement results from the latest clinical study will be published in a future report.

We suggest that these definitions be used as key features to avoid confusion and improve accuracy in suturing the prosthetic ring during repair of the area affected by earlyphase enlargement of the tricuspid annulus, and in tricuspid valve surgery.

\section{Conflict of Interest Statement}

Authors have nothing to disclose with regard to commercial support.

\section{References}

1. Nishimura RA, Otto CM, Bonow RO, Carabello BA, Erwin JP III, Guyton RA, et al. 2014 AHA/ACC guideline for the management of patients with valvular heart disease: a report of the American College of Cardiology/American Heart Association Task Force on Practice Guidelines. J Am Coll Cardiol. 2014;63: e57-185.

2. Cohn LH. Tricuspid regurgitation secondary to mitral valve disease: when and how to repair. J Card Surg. 1994;9(2 Suppl):237-41.

3. Kilic A, Saha-Chaudhuri P, Rankin JS, Conte JV. Trends and outcomes of tricuspid valve surgery in North America: an analysis of more than 50,000 patients from the Society of Thoracic Surgeons database. Ann Thorac Surg. 2013;96:1546-52; discussion 1552.

4. McCarthy PM, Bhudia SK, Rajeswaran J, Hoercher KJ, Lytle BW, Cosgrove DM, et al. Tricuspid valve repair: durability and risk factors for failure. J Thorac Cardiovasc Surg. 2004;127:674-85.

5. Ghanta RK, Chen R, Narayanasamy N, McGurk S, Lipsitz S, Chen FY, et al. Suture bicuspidization of the tricuspid valve versus ring annuloplasty for repair of functional tricuspid regurgitation: midterm results of 237 consecutive patients. J Thorac Cardiovasc Surg. 2007;133:117-26.

6. Sukmawan R, Watanabe N, Ogasawara Y, Yamaura Y, Yamamoto K, Wada N, et al. Geometric changes of tricuspid valve tenting in tricuspid regurgitation secondary to pulmonary hypertension quantified by novel system with transthoracic real-time 3-dimensional echocardiography. J Am Soc Echocardiogr. 2007;20: 470-6.

7. Fukuda S, Saracino G, Matsumura Y, Daimon M, Tran H, Greenberg NL, et al. Three-dimensional geometry of the tricuspid annulus in healthy subjects and in patients with functional tricuspid regurgitation: a real-time, 3dimensional echocardiographic study. Circulation. 2006;114(1 Suppl): I492-8.

8. Gatti G, Dell'Angela L, Morosin M, Maschietto L, Pinamonti B, Benussi B, et al. Flexible band versus rigid ring annuloplasty for functional tricuspid regurgitation: two different patterns of right heart reverse remodeling. Interact Cardiovasc Thorac Surg. 2016;23:79-89.

9. Silver MD, Lam JH, Ranganathan N, Wigle ED. Morphology of the human tricuspid valve. Circulation. 1971;43:333-48.

10. Carpentier A. Cardiac valve surgery: the "French correction" J Thorac Cardiovasc Surg. 1983;86:323-37.

11. Deloche A, Guerinon J, Fabiani JN, Morillo F, Caramanian M, Carpentier A, et al. Anatomical study of rheumatic tricuspid valve diseases: application to the study of various valvuloplasties [in French]. Ann Chir Thorac Cardiovasc. 1973;12:343-9.

12. Rogers JH, Bolling SF. The tricuspid valve: current perspective and evolving management of tricuspid regurgitation. Circulation. 2009;119:2718-25.

13. Athavale S, Deopujari R, Sinha U, Lalwani R, Kotgirwar S. Is tricuspid valve really tricuspid? Anat Cell Biol. 2017;50:1-6.

14. Seccombe JF, Cahill DR, Edwards WD. Quantitative morphology of the normal human tricuspid valve: autopsy study of 24 cases. Clin Anat. 1993;6: 203-12.

15. Victor S, Nayak VM. The tricuspid valve is bicuspid. J Heart Valve Dis. 1994;3: 27-36.

16. Pfannmüller B, Doenst T, Eberhardt K, Seeburger J, Borger MA, Mohr FW. Increased risk of dehiscence after tricuspid valve repair with rigid annuloplasty rings. J Thorac Cardiovasc Surg. 2012;143:1050-5.

17. Isomura T, Hirota M, Hoshino J, Fukada Y, Kondo T, Takahashi Y. Tricuspid annuloplasty with the MC3 ring and septal plication technique. Asian Cardiovasc Thorac Ann. 2015;23:5-10.

18. James TN. Anatomy of the crista supraventricularis: its importance for understanding right ventricular function, right ventricular infarction and related conditions. J Am Coll Cardiol. 1985;6:1083-95. 
19. Buckberg G, Hoffman JI. Right ventricular architecture responsible for mechanical performance: unifying role of ventricular septum. J Thorac Cardiovasc Surg. 2014;148:3166-71.

20. Ton-Nu TT, Levine RA, Handschumacher MD, Dorer DJ, Yosefy C, Fan D, et al. Geometric determinants of functional tricuspid regurgitation: insights from 3-dimensional echocardiography. Circulation. 2006;114: $143-9$.
21. Victor S, Nayak VM. Bicuspidization for tricuspid stenosis. Ann Thorac Surg. 1998;65:1468-70.

Key Words: tricuspid valve annuloplasty, subvalvular structure, right ventricular free wall, supuraventricular crest, commissural point 


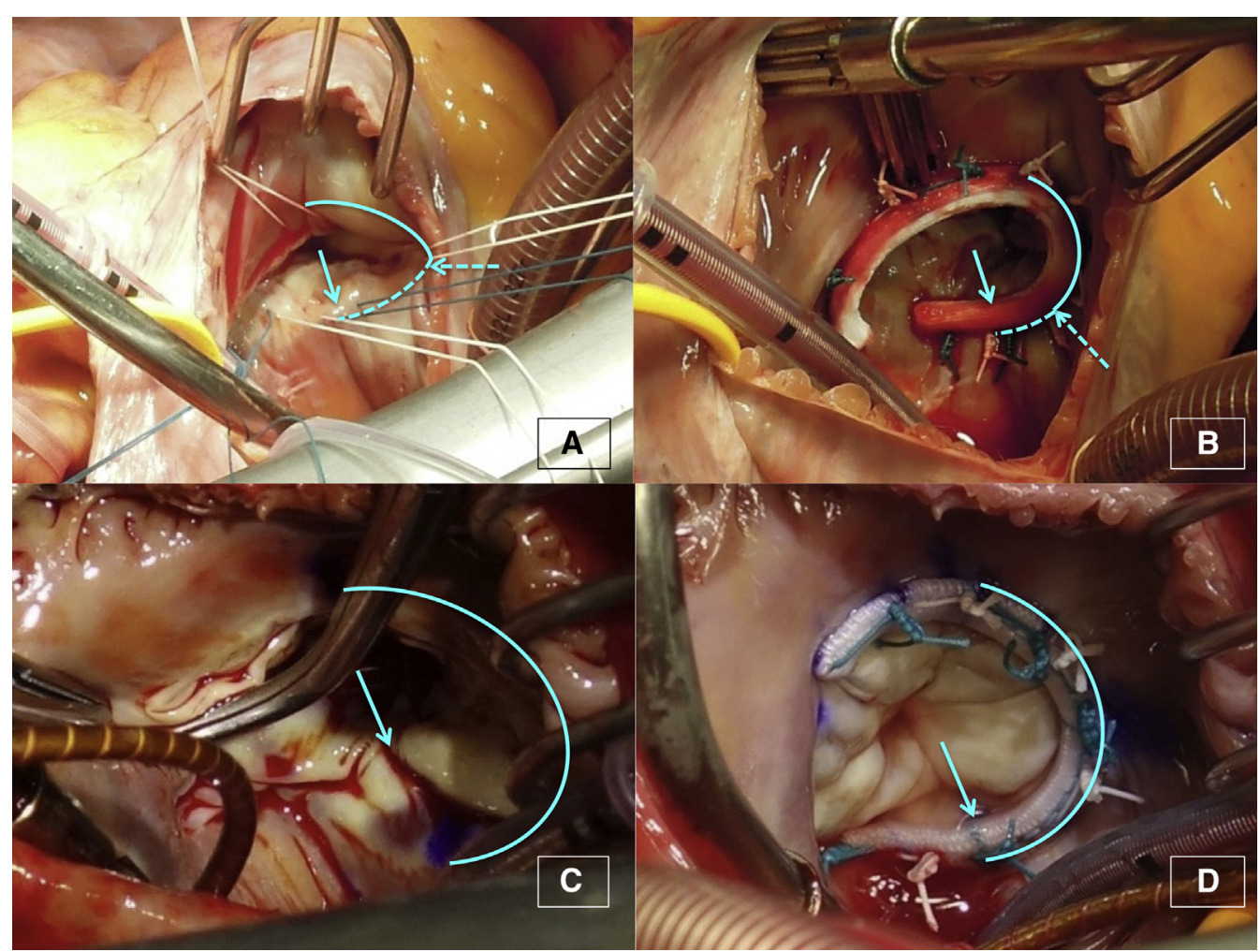

FIGURE E1. Completed tricuspid annuloplasty using the previous repair technique (A and B) and a currently described technique (C and D) showing significant differences, especially in the position of the posteroseptal commissure (PSC) area. Solid arrows indicate true predicted posteroseptal commissures (PSCPs); dashed arrows, false PSCPs.

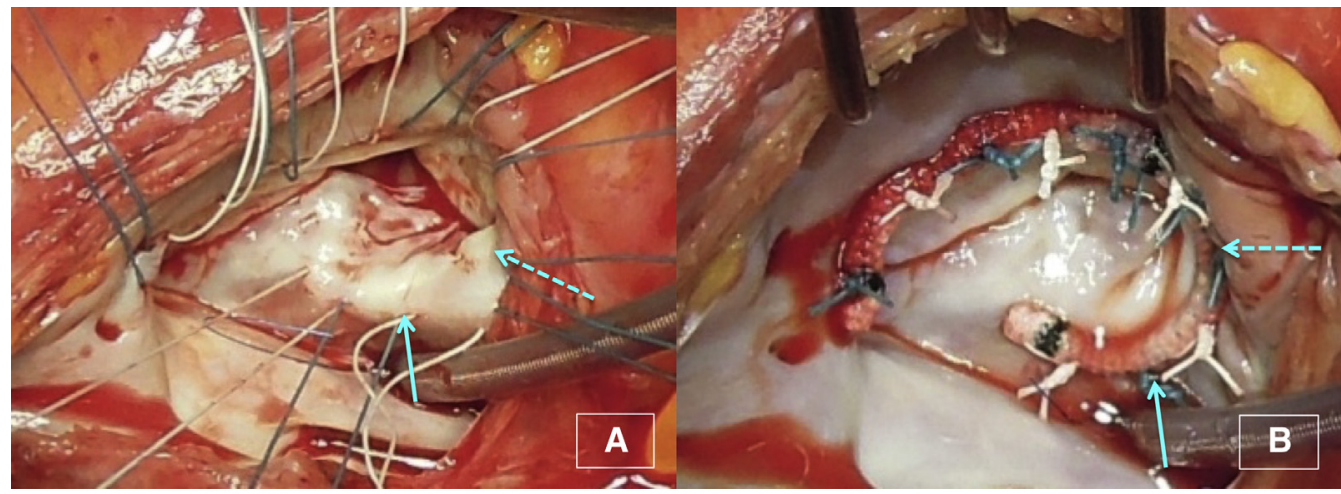

FIGURE E2. Misjudging the location of the posteroseptal commissure (PSC) when using a flexible band. A, Suture the mattress sutures through the tricuspid annulus around the orifice. B, Tricuspid valve after the flexible band has inplanted. The predicted PSCP is shown with dashed arrow, and true PSCP with solid arrow. The predicted posteroseptal commissure (PSCP) (dashed arrow) is located at the center of the posterior annulus. 